\title{
Implementing and Characterizing Precise Multiqubit Measurements
}

\author{
J. Z. Blumoff, ${ }^{1}$ K. Chou, ${ }^{1,}{ }^{*}$ C. Shen, ${ }^{1}$ M. Reagor,${ }^{1,2}$ C. Axline, ${ }^{1}$ R. T. Brierley, ${ }^{1}$ M. P. Silveri, ${ }^{1,3}$ \\ C. Wang, ${ }^{1}$ B. Vlastakis, ${ }^{1,4}$ S. E. Nigg,${ }^{5}$ L. Frunzio, ${ }^{1}$ M. H. Devoret,${ }^{1}$ L. Jiang, ${ }^{1}$ \\ S. M. Girvin, ${ }^{1}$ and R. J. Schoelkopf ${ }^{1}$ \\ ${ }^{1}$ Department of Applied Physics and Physics, Yale University, New Haven, Connecticut 06511, USA \\ ${ }^{2}$ Rigetti Quantum Computing, 775 Heinz Avenue, Berkeley, California 94710, USA \\ ${ }^{3}$ Research Unit of Theoretical Physics, University of Oulu, FI-90014 Oulu, Finland \\ ${ }^{4}$ IBM T.J. Watson Research Center, Yorktown Heights, New York 10598, USA \\ ${ }^{5}$ Department of Physics, University of Basel, Klingelbergstrasse 82, 4056 Basel, Switzerland \\ (Received 2 June 2016; revised manuscript received 26 July 2016; published 14 September 2016)
}

There are two general requirements to harness the computational power of quantum mechanics: the ability to manipulate the evolution of an isolated system and the ability to faithfully extract information from it. Quantum error correction and simulation often make a more exacting demand: the ability to perform nondestructive measurements of specific correlations within that system. We realize such measurements by employing a protocol adapted from Nigg and Girvin [Phys. Rev. Lett. 110, 243604 (2013)], enabling real-time selection of arbitrary register-wide Pauli operators. Our implementation consists of a simple circuit quantum electrodynamics module of four highly coherent 3D transmon qubits, collectively coupled to a high- $Q$ superconducting microwave cavity. As a demonstration, we enact all seven nontrivial subset-parity measurements on our three-qubit register. For each, we fully characterize the realized measurement by analyzing the detector (observable operators) via quantum detector tomography and by analyzing the quantum backaction via conditioned process tomography. No single quantity completely encapsulates the performance of a measurement, and standard figures of merit have not yet emerged. Accordingly, we consider several new fidelity measures for both the detector and the complete measurement process. We measure all of these quantities and report high fidelities, indicating that we are measuring the desired quantities precisely and that the measurements are highly nondemolition. We further show that both results are improved significantly by an additional error-heralding measurement. The analyses we present here form a useful basis for the future characterization and validation of quantum measurements, anticipating the demands of emerging quantum technologies.

\section{INTRODUCTION}

Building on impressive progress in control [1,2], measurement $[3,4]$, and coherence $[5,6]$, experimental quantum information science is addressing increasingly complex challenges, such as quantum error correction [7-12] and quantum simulation $[13,14]$. These applications frequently call for measurements of multiqubit properties, which can be qualitatively different from one-qubit measurements. Crucially, measurements of correlations, rather than complete state information, require a more refined concept of nondemolition. Strong single-qubit measurements project the system into a one-dimensional and trivial subspace, and nondemolition is guaranteed if repeated measurements agree. This is only a necessary, but not sufficient, condition

\footnotetext{
*kevin.chou@yale.edu
}

Published by the American Physical Society under the terms of the Creative Commons Attribution 3.0 License. Further distribution of this work must maintain attribution to the author(s) and the published article's title, journal citation, and DOI. for measurements of correlations, which must project the system into a multidimensional subspace while maintaining coherence within that subspace-an idea with no one-qubit analog. These measurements must be accomplished such that we learn only the desired information and no more. Great care is required to engineer this intricate interaction of a complex and delicate quantum system with the noisy and dissipative outside world.

In principle, these measurements can be constructed from a set of primitives consisting of one-qubit measurements and a universal set of one- and two-qubit gates. In practice, building up these circuits is not as simple as stringing these primitives together; decoherence and residual interactions play an increasingly important role. Residual interactions are of particular concern as they lead to correlated and coherent errors, can scale badly as additional qubits are added, and are potentially catastrophic for quantum error correction [15-17]. These challenges raise two questions: how do we design hardware and software to directly implement multiqubit measurements while addressing these concerns, and 
how do we reasonably characterize these measurements given different requirements?

Nondemolition multiqubit measurements have been implemented in a variety of architectures. Impressively, measurements of three- and four-qubit properties have been demonstrated in superconducting and ionic systems [18-21]. Quantification of these measurements has generally consisted of measurements of simple eigenstates of the intended measurement operator. Characterization of a multiqubit measurement process, including the backaction, has been reported for $Z Z$ measurements in two-qubit systems, via conditioned process tomography in superconducting qubits experiments [22,23]. Generally, the precise implementation of measurements within larger quantum systems is an important direction for further study. Superconducting qubits, our chosen platform, have strong electromagnetic interactions that lead to fast and high-fidelity control and single-qubit measurement. However, without care in engineering their environment, these same interactions can make these qubits vulnerable to cross talk and decoherence [11,23-25]. Among other adverse effects, this cross talk is also likely to pollute measurements (and through backaction, the system) with extraneous information.

With these issues in mind, in this work we demonstrate a novel 3D circuit quantum electrodynamics (cQED) architecture that exhibits direct qubit-qubit couplings significantly smaller than the qubit linewidths. Instead, interactions among our highly coherent and simple qubits are mediated by a common superconducting cavity. We use these interactions to engineer measurements of multiqubit properties via an ancilla qubit, adapting a proposal by Nigg and Girvin [26]. We demonstrate this protocol with a $3+1$ qubit system by performing all seven nontrivial three-qubit subset-parity measurements, $O_{i} \otimes O_{j} \otimes O_{k}: O_{x} \in\{I, Z\}$, excluding III. This set is of particular interest: when combined with single-qubit rotations, it generates the measurements of all possible product operators, which include those needed for stabilizer-based quantum error correction [8].

No single number fully characterizes a measurement, and the need for more sophisticated assessment is amplified for larger systems as they admit richer phenomena. Accordingly, we characterize each demonstrated measurement with three experiments and discuss several figures of merit. First, we perform an analysis similar to the measurement of computational states and report the assignment fidelity. Second, we introduce and employ a novel form of quantum detector tomography [27] to fully extract the positive operator valued measure (POVM) [28] that describes the realized detector. We do several analyses based on these results, introducing two fidelity measures and a complementary measure we call the specificity. Third, we consider the backaction induced by the measurement and reconstruct the measurement process maps
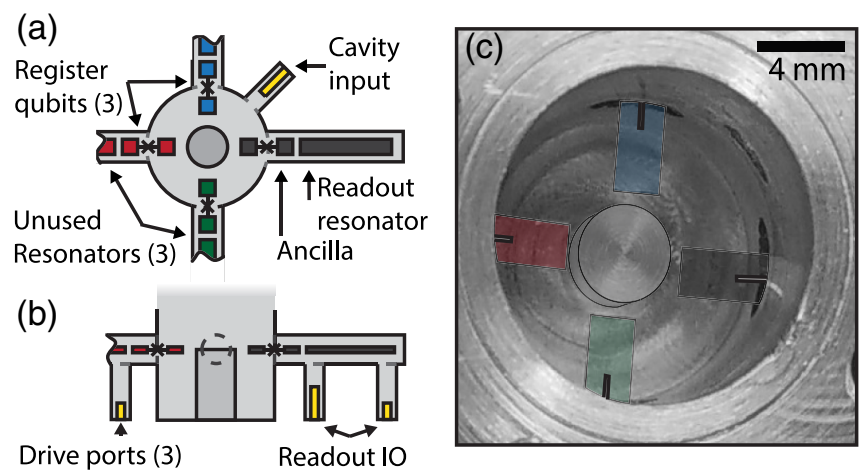

FIG. 1. The experimental sample consists of a central $\lambda / 4$ stub resonator [33], machined out of 6061 aluminum, with a lifetime of $72 \mu \mathrm{s}$, consistent with our expectation of the limitation due to surface losses. Four sapphire chips enter the cavity radially, each of which supports a 3D transmon and a quasiplanar coaxial $\lambda / 2$ resonator [34], patterned in the same lithographic step. All four $\lambda / 2$ resonators have undercoupled input ports for fast individual qubit control. One resonator has a low- $Q(1 / \kappa=60 \mathrm{~ns})$ output port that leads to a Josephson parametric converter (JPC) [3]. This enables high-fidelity (98\%) readout of the directly coupled qubit, which we designate as the ancilla. The other three qubits are designated as the register, and their associated three resonators are unused. All qubits share essentially identical capacitive geometry, but differing Josephson energies space the qubits by roughly $400 \mathrm{MHz}$. This results in dispersive shifts $\left\{\chi_{i}\right\}=$ $\{1.651,1.194,0.811,0.613\} \mathrm{MHz}$ and $\left\{\chi_{i j}\right\}$ generally on the order of $1 \mathrm{kHz}$. Further Hamiltonian and coherence details are in the Supplemental Material [32]. The cavity has an undercoupled input port, used for conditional and unconditional displacements, and a diagnostic output (which is also undercoupled and is not depicted). Panels (a) and (b) depict top view and side view schematics, respectively. Not to scale. (c) False-color top view of the physical device with outlines for clarity.

using conditioned process tomography. We further introduce two analogous fidelity measures for the measurement process. Additionally, many error mechanisms, such as relaxation, leave a distinctive signature, and we also report results heralded by an additional measurement confirming success. This may prove to be a useful feature, as heraldedsuccess gates can be efficiently used for universal quantum computation [29-31].

\section{IMPLEMENTING THE MEASUREMENT APPARATUS}

Our system is centered around a high- $Q$ superconducting cavity (hereafter, the "cavity" and with resonance frequency $f_{c}$ ), which is used mechanically as the isolating package for our module and quantum mechanically as an ancillary pointer state. Four 3D transmons (with $\mid g\langle\leftrightarrow \mid e\rangle$ transition frequencies $\left\{f_{i}\right\}$ ) couple to this cavity, with qubit-cavity dispersive interaction rates $\left\{\chi_{i}\right\}$ and qubitqubit longitudinal interaction rates $\left\{\chi_{i j}\right\}$. The simplified undriven Hamiltonian is given by 


$$
\begin{aligned}
H / h= & \sum_{i} f_{i}|e\rangle\left\langle\left. e\right|_{i}+f_{c} a^{\dagger} a-\sum_{i} \chi_{i} \mid e\right\rangle\left\langle\left. e\right|_{i} a^{\dagger} a\right. \\
& -\sum_{i, j \neq i} \chi_{i j}|e e\rangle\left\langle\left. e e\right|_{i j},\right.
\end{aligned}
$$

where we truncate the bosonic modes of the transmons to the two lowest energy levels. A more comprehensive Hamiltonian is given in the Supplemental Material [32]. Our device, depicted and described in Fig. 1, provides simultaneously strong qubit-cavity interactions and weak qubit-qubit interactions with $\chi_{i} / \chi_{i j} \approx 10^{3}$. This architecture also admits independent drive and readout channels, leading to minimal classical cross talk in both control and measurement. In this work, we utilize the readout channel (hereafter, the "readout resonator") of only one qubit, which serves as the ancilla. The other three qubits we collectively refer to as the register. Our protocol uses the qubit-cavity interactions to map a property of the register state (e.g., $Z Z Z$ or $Z Z I$ ) onto the ancilla via manipulation of the cavity state.

In the following, we provide a general overview of our three-step protocol. For more details, see Fig. 2. First, the cavity mode is displaced from the vacuum. It then acquires phase conditionally on the qubit states due to the dispersive interaction. This evolution is qualitatively akin to a continuous and parallel CPHASE interaction. As the cavity accumulates phase, we build the measurement operator qubit by qubit by using pairs of $X$ gates, analogous to a Hahn echo. If we want an $I$ in the measurement operator for a given qubit, i.e., not measuring it, we can perform a "full echo" decoupling sequence and completely average out the phase contribution of a particular qubit unconditionally (qubit 2 in Fig. 2). On the other hand, if we want a $Z$ in the measurement operator for a given qubit, then we want it to contribute a specific conditional phase angle to the cavity state. We may either allow the natural dispersive evolution to achieve this target conditional phase (qubit 1 in Fig. 2) or precisely tune this contribution by applying a "partial echo" sequence (qubit 3 in Fig. 2).

In that manner, measuring a qubit, or not, can be chosen by the timing of echoing gates. When all of the register qubits being measured give equal phase contributions, the phase of the cavity is a natural meter for the number of excitations in the measured subspace. This equalization can be considered as a stroboscopic erasure of which-path information, resulting in entanglement between a selected multiqubit property of the register and the phase of the cavity. The meter can go beyond excitation counting to map other operators; for example, if the accumulated phase per qubit is $\pi$, the cavity state measures register parity regardless of the size of the register. As this phase angle is set by the smallest dispersive shift of the measured qubits, we note that the total time of this protocol does not change as more qubits are added.

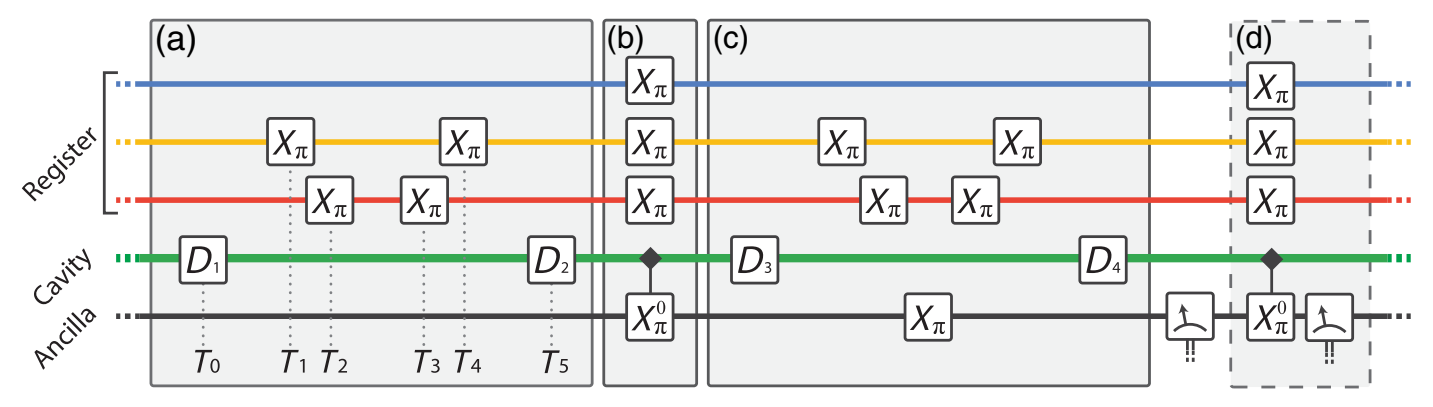

FIG. 2. Circuit diagram for $Z I Z$ measurement. Steps $X_{\pi}$ refer to one-qubit rotations around the $X$ axis by $\pi$ radians. Steps $X_{\pi}^{0}$ indicate that the pulses are spectrally narrow and are roughly selective on having zero photons in the cavity. Steps $D_{i}$ represent unconditional displacements of the cavity. The meters are measurements of the ancilla via the readout resonator, which is not itself depicted. The ancilla has the largest dispersive shift and the register qubits are then numerically ordered (from top to bottom) such that $\chi_{1}<\chi_{2}<\chi_{3}$. Prior to this procedure a series of measurements is applied to postselectively prepare the ground state; see the Supplemental Material for details [32]. (a) The algorithm begins with a displacement $D_{1}$ to create a coherent state of $\bar{n}=5$ photons into the cavity, which acquires a phase shift $\theta$ in a time $T=T_{5}-T_{0} \approx \theta /\left(2 \pi \chi_{1}\right)$ conditionally on the state of qubit 1 (blue line). For measurements of one- and two-qubit properties, $\theta=2 \pi / 5$. In this example, we perform a full echo on the second qubit (yellow line) by performing two unconditional $X$ gates separated in time by $T_{4}-T_{1} \approx T / 2$. The third qubit (red line) would contribute a conditional phase shift of $2 \pi \chi_{3} T>\theta$. We reduce this to $\theta$ by performing two $X_{\pi}$ gates separated by $T_{3}-T_{2} \approx \theta\left(\chi_{1}^{-1}-\chi_{3}^{-1}\right) / 2$. At $T_{5}$, we perform $D_{2}$ to shift the odd two-parity coherent-state pointer to the zero-photon state. Note that the overlaps between the even two-parity pointer states and the zero-photon state are exponentially suppressed. (b) We map this photon number information onto the ancilla qubit with a $X_{\pi}^{0}$ gate, taking advantage of the well-known number-splitting phenomenon [35]. As the cavity states are separated by $\approx 6.5$ photons, we employ a faster, approximately selective gate, $300 \mathrm{~ns}$ in duration. $X_{\pi}$ gates on the register are centered on this pulse in time to echo away the cavity evolution during this step. (c) To disentangle the cavity pointer states, we essentially invert the pulse sequence of (a), returning the cavity to the vacuum state. We must also echo the ancilla, as it may now be excited. This results in a total gate length of 970 ns. Subsequently, we measure the ancilla qubit. (d) This optional step determines if there are residual photons in the cavity. Since many types of errors result in residual photons, a subsequent photon-number-selective rotation and measurement of the ancilla heralds these errors. When measuring three qubits (e.g., ZZZ), we choose $\theta=\pi$, so that the cavity states entangled with the one- and three-excitation manifolds recohere. 
In the second step [Fig. 2(b)], the ancilla qubit samples whether or not the cavity has acquired some chosen phase. An unselective displacement shifts one of the cavity states to the vacuum, converting phase information into photon number-state information. A spectrally narrow pulse then excites the ancilla if and only if there are zero photons in the cavity. These first two steps are a natural multiqubit extension of the ideas used in the qcMAP gate [36], which maps the state of the transmon qubit onto the phase of a coherent state in a harmonic oscillator. With the chosen property of the register now imprinted onto the ancilla state, we could measure the ancilla directly, but if we hope for the measurement to be nondestructive, we must first disentangle the cavity. In the last step [Fig. 2(c)], we remove this residual entanglement, unconditionally resetting the cavity to the vacuum, by essentially inverting (or "echoing") the unitary dynamics of the first step. Finally, we use this composite gate to enact a multiqubit measurement by interrogating the readout cavity, which is sensitive only to the ancilla state.

We optionally append an additional manipulation and measurement to verify that the cavity has been reset to the vacuum [Fig. 2(d)]. This condition is not achieved when we have experienced errors due to qubit or cavity relaxation, as well as certain effects from higher-order terms in the Hamiltonian. While our implementation utilizes a 3D circuit QED system, the protocol is more general, relying only on common dispersive shifts to a harmonic oscillator. Like any precise operation in a large Hilbert space, it also benefits significantly from high coherence and low residual couplings, as achieved in our device. Any implementation that can realize a similar Hamiltonian is also suitable for this measurement protocol.

\section{MEASUREMENT CHARACTERIZATION}

With these measurements manufactured, we turn to the problem of describing them quantitatively. This endeavor does not have a one-size-fits-all resolution: different applications have differing needs, requiring experiments and analyses of differing experimental complexity in the system dimension $d$. Accordingly, we attempt to anticipate many potential desiderata, and perform several analyses on the results of three separate experiments. The first two experiments examine the detector alone, neglecting backaction on the input state, and the third goes on to examine the measurement process entirely.

The first and simplest analysis provides a partial characterization of the detector requiring $\mathcal{O}(d)$ data points. In Sec. III A, we explore assignment fidelity: How much desired information are we getting?

We expand on this with a full characterization of the detector and extract several figures of merit $\left[\mathcal{O}\left(d^{2}\right)\right.$ data points]. Section III B examines quantum detector tomography: What is the POVM that describes our detector? What are we actually learning? In Sec. III C, we present specificity: What is the maximal measurement contrast along any axis? And a complementary question: How close is this maximal axis to the desired axis? In Sec. III D, we at look at detector fidelity: How close is our POVM to the desired measurement?

We additionally may care a great deal about the backaction of the measurement, a consideration crucial for stabilizer-based quantum error correction. For this, we perform a third experiment to characterize the measurement process and extract pertinent figures of merit based on the quantum instrument formalism [37-39] $\left(\mathcal{O}\left(d^{4}\right)\right.$ data points). In Sec. III E, we present measurement process tomography: What makes the detector click and what happens to the state after measurement? And in Sec. III F, we examine quantum instrument fidelity: How close are the measurement processes, inclusive of backaction, to the desired measurement?

As the quantum instrument encompasses the detector, a discrepancy between the quantum instrument and detector fidelities provides an assessment of the undesired backaction on the system.

In order to extract the detector and quantum instrument fidelities, we describe our measurements as channels that introduce the detector as a classical state in an additional Hilbert space. We provide more details on this interpretation as these quantities are introduced. This treatment allows us to appropriate commonly used figures of merit for quantum processes and apply them to measurements. Following the reasoning in Gilchrist et al. [40], for each analysis we report the two sets of measures: first, the $J$ fidelity, which is derived directly from the channel Jamiołkowski matrix, and second, the $S$ fidelity, which is a conservative measure based on the worst-case input state. The $J$ fidelities are similar to measures given in Refs. [41,42]. We provide the ranges for all of these results in the main text, and provide tables of the full results in the Supplemental Material [32]. In addition to the fidelities, we also provide the analogous $J$ and $S$ distances, the latter of which is commonly called the diamond distance $[28,43]$.

\section{A. Assignment fidelity}

A simple way to define the performance of a binary measurement is to assume the model: i.e., how sensitive is our measurement to the desired quantity (e.g., ZIZ)? To answer this, we prepare states of known ideal measurement outcome, measure, and fit to find the correlation between the state preparation and experimental outcomes. This yields a contrast and an offset, which can also be interpreted to tell how often we get the expected result. As an example, for a simple $Z$ measurement of a qubit, it is common to prepare the computational $(|0\rangle$ and $|1\rangle)$ states and to report how often the measurement outcomes agree with the state preparation. This is often referred to as assignment fidelity. The extension to multiqubit subset-parity measurements typically involves preparations of $d$ computational states; see, e.g., Ref. [19]. 
(a) $Z Z Z$
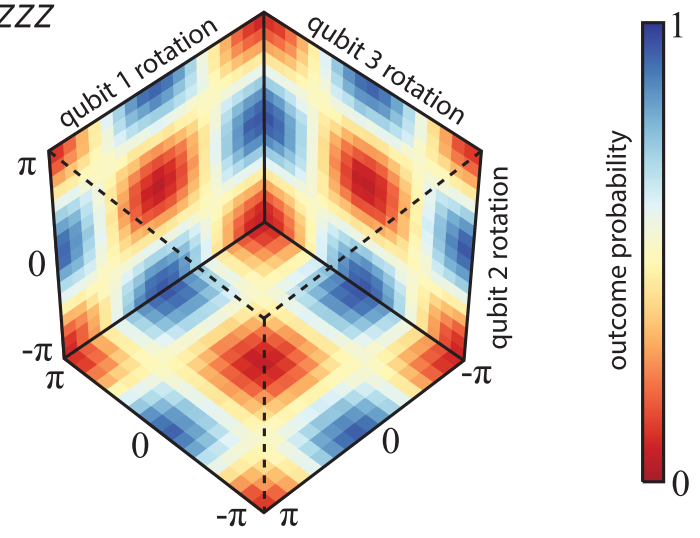

(b)

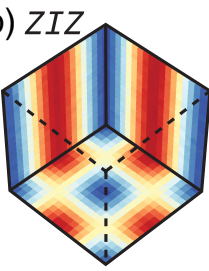

(c) $Z Z I$

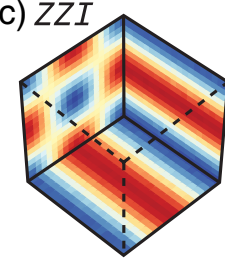

(d) $I Z Z$

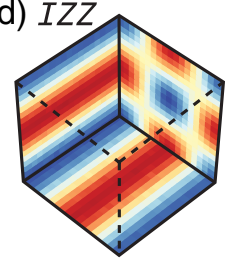

(e) $Z I I$

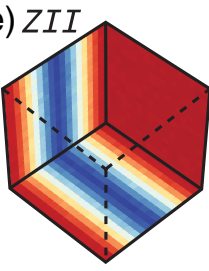

(f) $I Z I$

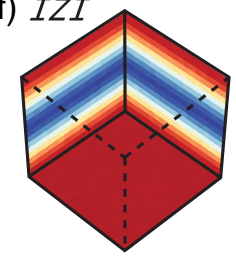

(g) IIZ

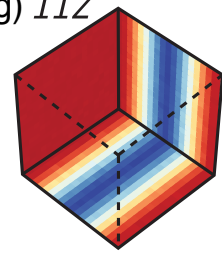

FIG. 3. Demonstration of parity measurement outcomes. For all seven nontrivial three-qubit subset-parity operators, we show ancilla excitation probabilities as a function of initial register state. In each panel, the three axes specify the initial state of each register qubit, parametrized by rotation angle about the $X$ axis after initialization in the ground state. We depict three plane cuts through that parameter space. The color scale indicates the probability to find the ancilla in the excited state. Panel (a) shows measurement operator ZZZ. The second row (b)-(d) shows twoqubit parity measurements. It is easily seen that the outcome is independent of the preparation of one qubit. The third row (e)- $(\mathrm{g})$ shows single-qubit measurements, reflecting sensitivity to only one preparation axis. We extract assignment fidelities from these data of $89 \%-95 \%$ that improve to $94 \%-97 \%$ with postselection on a success herald.

We show the results for our measurements with a similar but slightly more illustrative experiment in Fig. 3. We prepare a larger number of states than required, but the result is essentially the same as we fit the data to our expected correlation. We find contrasts well over $90 \%$ for most of our measurements, and in some cases approach the limit set by our ancilla readout, showing that our detectors are highly sensitive to the operator we expected. Assignment fidelity is a useful diagnostic tool as it is quickly measured and has a simple interpretation; however, it provides limited information. As an exaggerated example, if you expected a $Z$-sensitive detector, this present analysis would give identical results if used to examine

either a random number generator or a perfect $X$-sensitive detector. Errors like the latter, if undiscovered, would result in misleading, skewed state estimation, but are correctable with unitary control. A simple noisy reduction of contrast has neither of these properties.

\section{B. Quantum detector tomography}

More generally, we may ask of a binary-outcome detector: for what inputs does it "click"? In quantum mechanics we describe these detector-outcome probability distributions with the POVM formalism. The measurement is represented as a set of operators $\left\{E_{i}\right\}$ with the probability of measurement outcome $i$ equal to $\operatorname{Tr}\left[E_{i} \rho\right]$ given an input state $\rho$. As POVMs are complete, we may fully describe a binary POVM with one operator: $\left\{E_{0}, E_{1}\right\} \equiv\{E, I-E\}$.

To characterize the detector more rigorously, we prepare a complete or overcomplete set of known input states and record the measurement outcome distribution, or click probability. We reconstruct $E$ from these data with a linear inversion. This procedure is called quantum detector tomography [27,44]. It is essentially identical to traditional state tomography, differing only in the prior assumptions: rather than assuming we know the measurement operator, as we do in state tomography, we assume knowledge of the input state. This knowledge is imperfect, but we note that our ground state preparation is better than $99 \%$, and our one-qubit gate errors are less than $0.2 \%$, as determined from randomized benchmarking [45]. Our implementation of detector tomography is the first that we know of outside of photonic experiments for system dimensions greater than two, and the first we know of at all in superconducting systems. We also note that a weaker, diagonal form of detector tomography that assumes sensitivity only to $I$ and $Z$ correlations, akin to our assignment fidelity analysis, is often implicitly used to correct for measurement errors in state tomography [46].

For the seven demonstrated measurements, $\{Z Z Z, Z Z I$, $Z I Z, I Z Z, Z I I, I Z I, I I Z\}$, we reconstruct the relevant measurement operators, which are ideally projectors onto a subspace of definite measurement outcome. Several examples are shown in Fig. 4, and we see that the results are close to the expected operators. We can also distill this full measurement operator into more easily interpretable figures of merit.

\section{Specificity}

What is the maximum information our measurement gains about any quantity? Or from another point of view: Is our detector infidelity due to noise and simple lack of contrast, or is it because our detector is measuring the wrong quantity? Furthermore, is our detector biased-given a completely mixed input state, is one measurement outcome more likely than another? This analysis is general to all strong binary measurements, but here we assume for simplicity that the ideal measurement is of a target Pauli operator $\sigma_{T}$. 


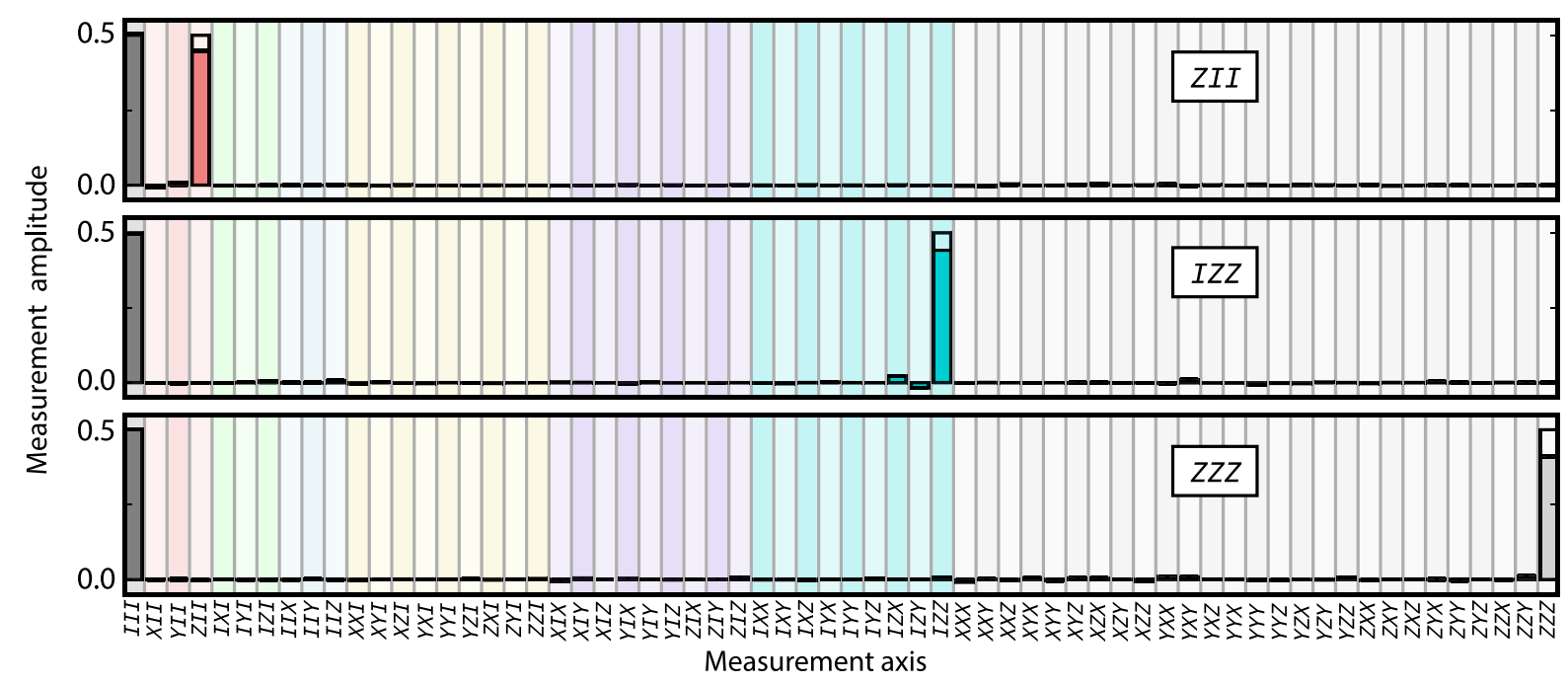

FIG. 4. Results of quantum detector tomography for three selected operators, using the unheralded data sets. We expand the first element $E$ of each POVM in three-qubit generalized Pauli operators $\sigma_{i}$, so that $E=\sum_{i} c_{i} \sigma_{i}$, and show the magnitudes of the coefficients of that expansion. For measurement of a Pauli operator, each should have two nonzero bars (amplitude 0.5 ) corresponding to the identity and the operator of the measurement, $\sigma_{m}$. Deviations of the identity bar from 0.5 indicate that the meter has some bias in the detectoroutcome distribution. When the amplitude of the $\sigma_{m}$ bar is less than 0.5 , it indicates the measurement does not have full contrast along the desired axis. Finite values of the other bars indicate that our measurement has undesired sensitivity to an extraneous property. The POVM $J$ fidelities for the illustrated operators are $95 \%, 94 \%$, and 91\%, respectively. The other four realized measurement operators, as well as reconstructed POVMs from the success-heralded data set, are provided in the Supplemental Material [32].

We express $E$ in the Pauli basis (which fully spans the space of $n$-qubit observables), as in Fig. 4, leading to a vector of Pauli coefficients. One dimension corresponds to the identity axis, one to the desired operator, and the rest to the various other Pauli operators. We can then find a new basis that rotates this vector space, leaving the identity and $\sigma_{T}$ axes invariant, such that only one other nonzero coefficient in the measurement vector remains. This additional coefficient $c_{O}$ corresponds to an orthogonal rotated Pauli $\sigma_{O}$,

$$
E=c_{I} I+c_{T} \sigma_{T}+c_{O} \sigma_{O}
$$

The coefficients of this expansion are easily interpreted: a deviation of $c_{I}$ from the ideal value of 0.5 describes the bias of the detector, $2 c_{T}$ represents the maximum possible gain of information about the quantity we wish to measure, and $2 c_{O}$ represents the magnitude of the potential undesired information gain. Note that $E$ must be a positive matrix, which yields constraints on $c_{T}$ and $c_{O}$ relative to the bias term $c_{I}$; e.g., a detector that always clicks cannot yield useful information. The infidelity corresponding to measuring along the wrong axis can be considered as analogous to coherent errors for standard processes, since a unitary rotation of the system prior to measurement would remove it. This analysis prescribes this correcting unitary exactly. In some cases the correction may be separable into singlequbit operations, but will not be true in general, particularly for architectures with high residual couplings.
This formulation leads naturally to a description in terms of angles between vectors, and we can quantify how well we are measuring along the correct axis with an angle for the "specificity" of the measurement, $\theta_{s} \equiv \arctan \left(c_{O} / c_{T}\right)$. We find our measurements to be within $1^{\circ}-5^{\circ}$ of the target operator, indicating that our measurements are not yielding significant information about unwanted quantities. In addition, we see that our detectors have very little bias.

Returning to the initial question of maximal information gain, we recast Eq. (2) as $E=c_{I} I+c_{\max } \sigma_{\max }$, where the operator $\sigma_{\max }$ is defined as the axis where the measurement gains the most information. The coefficient $c_{\max }=\left(c_{T}^{2}+\right.$ $\left.c_{O}^{2}\right)^{1 / 2}$ directly quantifies the total information gain along this axis. As might be expected from our small $\theta_{s}$, we find $c_{\max } \approx c_{T}$ for our measurements.

\section{Detector fidelity}

Generally, how similar are two detectors? We extend this standard POVM formalism by describing the detector as a quantum channel [37]. This detector channel takes a quantum state of the register $\rho_{r}$ as input and yields a diagonal density operator $\rho_{d}$ with entries that represent the detector-outcome probabilities,

$$
\mathcal{E}_{\mathrm{det}}: \rho_{r} \mapsto \rho_{d}=\sum_{i} \operatorname{Tr}\left[E_{i} \rho_{r}\right]|i\rangle\left\langle\left. i\right|_{d} .\right.
$$

The output state of the detector $\rho_{d}$ is represented as a density operator but should be understood as a container for 
a purely classical probability distribution. It represents the recorded measurement outcome, not the state of our physical ancilla transmon. The detector channel $\mathcal{E}_{\text {det }}$ is a completely positive trace-preserving map that can be described with nonsquare Kraus operators. The channels relevant to the present experiment act on an eightdimensional (three-qubit) register and yield a twodimensional (binary-outcome) classical detector state.

We compare the experimental detector channels to the ideal processes with two figures of merit. We start with the $J$ fidelity,

$$
\mathcal{F}_{J}\left(\mathcal{E}^{(1)}, \mathcal{E}^{(2)}\right) \equiv \mathcal{F}_{\operatorname{Tr}}\left(\boldsymbol{J}^{(1)}, \boldsymbol{J}^{(2)}\right),
$$

where $J^{(i)}$ is the Jamiołkowski matrix representing the process $\mathcal{E}^{(i)}$ and $\mathcal{F}_{\operatorname{Tr}}(\rho, \sigma) \equiv\left(\operatorname{Tr} \sqrt{\rho^{1 / 2} \sigma \rho^{1 / 2}}\right)^{2}$. For our measurements, we calculate detector $J$ fidelities between $91 \%$ and $95 \%$, which improve to $95 \%-98 \%$ with postselection on the success herald. This error detection is efficient as the fraction of experiments discarded is similar to the improvement in fidelity. These measurements approach the limit set by our ancilla readout, $98 \%$. The detector $J$ fidelity can be similar to assignment fidelity, but is more general. It is applicable to measurements with more than two outcomes and allows for comparison of less than full-contrast operators. Unlike some comparable figures of merit, detector $J$ fidelity does not require renormalization of the POVM operators, which may discard information.

For standard nonmeasurement processes, the worst-case performance is often more important than the $J$ fidelity, and accordingly, we also report the $S$ fidelity [40]. This measure provides a conservative bound on the detector performance, including any possible degradation when the measurement is applied to a subspace within a larger, entangled quantum system. This $S$ fidelity applied to the detector process is given by

$\mathcal{F}_{\mathrm{det}}\left(\mathcal{E}_{\mathrm{det}}^{(1)}, \mathcal{E}_{\mathrm{det}}^{(2)}\right) \equiv \min _{\rho_{r a}} \mathcal{F}_{\mathrm{Tr}}\left[\mathcal{E}_{\mathrm{det}}^{(1)} \otimes I\left(\rho_{r a}\right), \mathcal{E}_{\mathrm{det}}^{(2)} \otimes I\left(\rho_{r a}\right)\right]$,

where $\rho_{r a}$ represents a joint pure state $|\psi\rangle\left\langle\left.\psi\right|_{r a}\right.$ of the register and an ancillary, potentially entangled space, e.g., the rest of a quantum computer. We emphasize that the states resulting from the process and compared on the righthand side are states of the detector and ancillary space. In the special case of two-outcome POVMs, we believe the state that reveals the worst-case performance will always be separable, indicating that $\mathcal{F}_{\text {det }}$ is inherently stable. In the Supplemental Material [32], we give a proof of this in the case of the two-outcome detector $S$ distance, and we have numerical evidence suggesting that the two-outcome detector $S$ fidelity has the same property. We stress that the minimization is still useful, as it yields the worst-case performance. Additionally, it can be easily shown that the $\mathcal{F}_{\text {det }}$ reduces to a minimization of the (square of the) classical fidelity of the probability distribution of the detector outcomes. The minimization over input states is performed using a semidefinite programming package in MATLAB [47,48]. With this measure, the seven detector fidelities we report are between $88 \%$ and $95 \%$, which improve to $93 \%-97 \%$ with postselection on the success herald. We see that for our realized detectors the $J$ fidelities are $1 \%-3 \%$ better than the worst-case performance.

\section{E. Measurement process characterization}

We have now characterized the behavior of the detector, but what happens to the input state after a measurement result is recorded? What is the backaction of registering a click or no click? Quantum error correction, for example, demands that the measurements must be highly quantum nondemolition in the sense of a von Neumann measurement. When an ancilla measurement indicates an outcome, e.g., that the register has positive $Z I Z$, the quantum process performed is ideally a projector onto the specified subspace. One method of analysis is to describe this process by two trace-nonpreserving maps on the register Hilbert space, $\left\{F^{0}, F^{1}\right\}$. We quantify these maps by performing outcomedependent quantum process tomography, which has been previously demonstrated for two-qubit measurements $[22,23]$.

This reconstruction begins by preparing a complete set of initial register states. For each initial state, we perform our measurement, record the outcomes, and perform state tomography conditioned on those outcomes. We employ a maximum likelihood estimation (MLE) for each state tomogram, then weight the outcome states by the probability that each measurement outcome is observed. This subtlety leads to individually trace-nonpreserving maps. To extract the process, we then perform an additional MLE relating the input states to both sets of output states, constraining the full measurement channel to be positive and trace preserving. It would be preferable to perform a single MLE, rather than two, but this problem is computationally imposing in the three-qubit case. The sequential approach has the additional benefit of allowing us to recalibrate drifts in our tomographic measurement operator throughout the several hours of data acquisition.

The reconstructed conditioned maps for the $Z Z Z$ operator are partially shown in Fig. 5. Ideal measurements of generalized Pauli operators have four real elements in the $\chi$ matrix representation. Each of these has amplitude 1/4, with positive diagonal elements and off-diagonal elements that change sign between the two outcomes. We find good qualitative agreement between this and our experimental data with a small decrease in contrast, indicating that the backaction is close to the ideal von Neumann projections. 

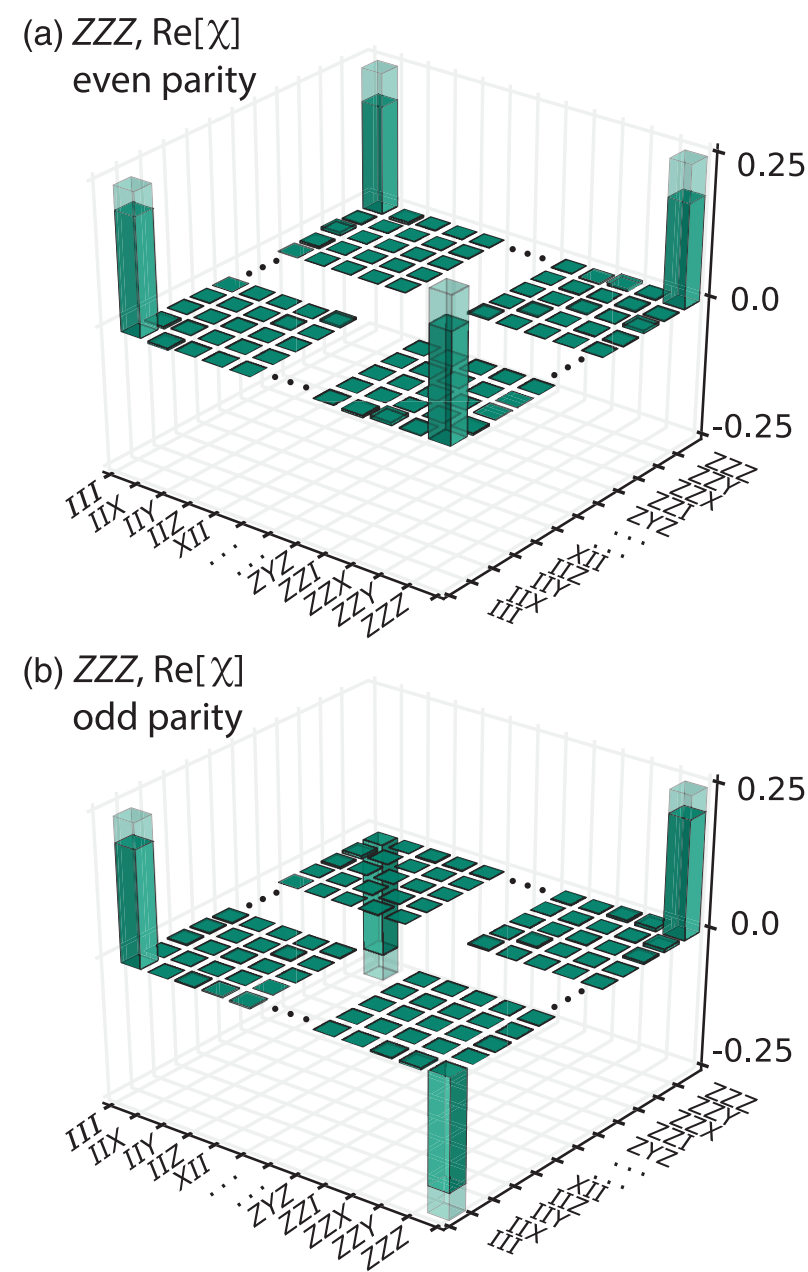

FIG. 5. Three-qubit conditioned quantum process tomography. Experimental quantum process tomography results for (a) even and (b) odd outcome process maps for the three-parity measurement, ZZZ. We express our process tomography in the Pauli basis where the conditioned processes can be described using $\chi$ matrix notation: $F^{0(1)}\left(\rho_{r}\right)=\sum_{i j} \chi_{i j}^{0(1)} \sigma_{i} \rho_{r} \sigma_{j}$, where $\{\sigma\}$ are the threequbit generalized Pauli operators. Here, we show only the corners of these process matrices, all other parts are visually indistinguishable from noise. Data on the full reconstruction, including of the other six measurement operators, are given in the Supplemental Material [32]. The ideal even and odd outcome processes are projectors $\Pi^{0(1)}=(I I I \pm Z Z Z) / 2$, and the corresponding $\chi$ matrices have a simple form consisting of only four real components in the generalized Pauli basis, and this ideal form is overlaid with wire frame bars. Note that we plot only the real components as all experimental imaginary components are visually indistinguishable from noise. We calculate the $J$ fidelity (as defined in Sec. III F) for this operator to be $80 \%$.

\section{F. Quantum instrument fidelity}

How can we quantitatively compare the performance of our experimentally reconstructed measurement process to the ideal measurement? We answer this question by representing the full measurement process as a quantum instrument [37], an approach that parallels our previous treatment of POVMs as channels and shares many of its advantageous properties. A quantum instrument describes a single channel that takes in a quantum state of the system and outputs both a quantum state of the system as well as a detector-outcome state. This detector-outcome state is the same as in Eq. (3) and signals the conditioned backaction induced on the system input state. For a two-outcome measurement, the quantum instrument can be written as

$$
\begin{aligned}
\mathcal{E}_{\mathrm{QI}}: & \rho_{r} \mapsto F^{0}\left(\rho_{r}\right) \otimes \rho_{d}^{0}+F^{1}\left(\rho_{r}\right) \otimes \rho_{d}^{1}, \\
& \stackrel{\text { ideal }}{\mapsto} \Pi^{0} \rho_{r} \Pi^{0} \otimes \rho_{d}^{0}+\Pi^{1} \rho_{r} \Pi^{1} \otimes \rho_{d}^{1},
\end{aligned}
$$

where $\rho_{r}$ refers to the input state in the register space, $\rho_{d}^{0(1)}$ refer to diagonal states in the detector subspace $\left(|0\rangle\left\langle\left. 0\right|_{d}, \mid 1\right\rangle\left\langle\left. 1\right|_{d}\right)\right.$, and $\Pi^{0(1)}$ are projectors in the register space onto orthogonal measurement outcomes. Similar to our previous analysis of the detectors (without backaction), we derive fidelity measures for these quantum instrument channels. The $J$ fidelities for quantum instrument channels follow from Eq. (4) with the appropriate quantum instrument maps and are calculated between $67 \%$ and $75 \%$. These improve to $79 \%-83 \%$ with postselection on the success herald. We also report the $S$ fidelity for these channels,

$$
\mathcal{F}_{\mathrm{QI}}\left(\mathcal{E}_{\mathrm{QI}}^{(1)}, \mathcal{E}_{\mathrm{QI}}^{(2)}\right) \equiv \min _{\rho_{r a}} \mathcal{F}_{\mathrm{Tr}}\left[\mathcal{E}^{(1)} \otimes I\left(\rho_{r a}\right), \mathcal{E}^{(2)} \otimes I\left(\rho_{r a}\right)\right]
$$

following the same notational caveats as Eq. (5).

For our experimental results, we calculate $\mathcal{F}_{\mathrm{QI}}$ of $57 \%-64 \%$, which increase to $69 \%-76 \%$ with postselection on the success herald. We see that the worst-case performance is as much as $10 \%$ worse than the $J$-fidelity measure. Note that for the success-heralded data, we include the requisite selective rotation and measurement in the definition of the process, which exposes the register to another $1.5 \mu \mathrm{s}$ of decoherence, though we do employ a Hahn echo on all qubits in the register. This check could be made significantly faster with an additional, dedicated qubit with a large dispersive shift.

\section{DISCUSSION}

We show that the implemented multiqubit measurements behave as intended: they are highly specific to the desired operator and have detector fidelities that approach the bound set by our single-qubit measurements. Additionally, we see that the backaction of the measurement is indeed close to the ideal, but, unsurprisingly, it is worse than our measurement contrast.

The performance we demonstrate here is limited by several effects. The largest source of infidelity for both the detectors and processes is cavity photon loss, which can be greatly reduced by moving to high-purity etched 
aluminum. This is known to give a factor of 10-20 improvement in quality factor in similar systems [33], and in similar samples has also increased qubit relaxation times [49]. The second-largest imperfection in the process performance is the dephasing of one of the register qubits, which displays a significant low-frequency beat in Ramsey experiments during this experimental run. Both detector and process suffer from the low $T_{1}$ of only $20 \mu$ s for the ancilla, which is limited by the Purcell effect and which may be improved with a Purcell filter [34]. The next-largest imperfections for the detector performance are the relaxation rates of the register qubits. Additional significant sources of error are the finite cavity anharmonicity and the difficulty in doing photon-number unconditional $X$ gates when photons are present. These two effects, as well as other coherent errors, may be circumvented by engineering a sequence via optimal control techniques [50], which is feasible for small modules that have weak interactions with their environment. The process $J$ fidelities we obtain are consistent with numerical simulations [51] that include all known error sources.

We are unable to directly compare our results to preexisting results in the field, but the most related experimental work [22,23] and theoretical work [41,42] cite fidelity measures that are similar to our quantum instrument $J$ fidelity (or its square root). We reiterate that our quantum instrument $J$ fidelities are as much as $10 \%$ higher than our quantum instrument $S$ fidelities. In many cases, the worst-case performance is of greater importance, as in fault-tolerance considerations. Indeed, we believe that it may be interesting to incorporate $\mathcal{F}_{\mathrm{QI}}$, or the analogous quantum instrument diamond distance, into threshold calculations, since it directly provides a conservative performance estimate of the operation central to stabilizer-based error correction. As a more "compiled" operation, the full measurement may be more sensitive to nonidealities than concatenated fidelity estimates of smaller one- and two-qubit operations.

A more traditional $Z Z Z$ measurement would consist of three CNOT gates and a single-qubit measurement of an ancilla. Measures derived from the quantum instrument not only take the performance of those simple operations into account, but also account for negative effects due to decoherence and residual interactions among the rest of the qubits. This includes the duration of the ancilla measurement, which by itself often has unintended and detrimental effects on other qubits in some systems. The work of incorporating $\mathcal{F}_{\text {QI }}$ into threshold calculations is not within the scope of this result; however, we believe that calculation of thresholds that are more inclusive of experimentally observed nonidealities may become a useful area of investigation. Though this analysis requires reconstruction of the process, it may not be overly burdensome for several-qubit stabilizers if compressed sensing techniques are employed [52].

\section{CONCLUSION}

We demonstrate a versatile quantum gate ideally suited to our highly coherent $3 \mathrm{D} c \mathrm{QED}$ architecture and use it to enact high-fidelity and specific multiqubit measurements. It is possible to realize strong interactions between fixedtuned qubits in this system, despite having low direct couplings, and there are clear pathways to further improved performance. As quantum systems continue to grow in complexity, it will be crucial to build systems with low cross talk and residual interactions like the one we demonstrate here. We also present several new approaches to analyze and characterize measurements, including two new conservative measures to quantify the fidelity of detectors and measurement processes. Complex measurements within larger systems will become increasingly important, and the figures of merit we introduce in this work may prove to be useful tools to benchmark their performance.

\section{ACKNOWLEDGMENTS}

We acknowledge Reinier Heeres and Philip Reinhold for helpful discussions and software assistance. We acknowledge Katrina Sliwa, Anirudh Narla, and Michael Hatridge for providing our JPC and advising us on its installation and use. We acknowledge Wolfgang Pfaff, Haidong Yuan, and Victor Albert for helpful discussions. Facilities use was supported by the Yale SEAS clean room, YINQE, and NSF MRSEC DMR-1119826. This research was supported by the Army Research Office under Grant No. W911NF-14-10011 and by Office for Naval Research under Grant No. FA9550-14-1-0052. C. A. acknowledges support from the NSF Graduate Research Fellowship under Grant No. DGE-1122492. S. M. G. acknowledges additional support from the NSF under Grant No. DMR-1301798. M.P. S. acknowledges additional support from the Alfred Kordelin Foundation. S. E. N. acknowledges support from the Swiss NSF.

J.Z.B. and K. C. contributed equally to this work.

[1] R. Barends et al., Superconducting Quantum Circuits at the Surface Code Threshold for Fault Tolerance, Nature (London) 508, 500 (2014).

[2] J. P. Gaebler, T. R. Tan, Y. Lin, Y. Wan, R. Bowler, A. C. Keith, S. Glancy, K. Coakley, E. Knill, D. Leibfried, and D. J. Wineland, High-Fidelity Universal Gate Set for ${ }^{9} \mathrm{Be}^{+}$ Ion Qubits, Phys. Rev. Lett. 117, 060505 (2016).

[3] N. Roch, E. Flurin, F. Nguyen, P. Morfin, P. CampagneIbarcq, M. H. Devoret, and B. Huard, Widely Tunable, Nondegenerate Three-Wave Mixing Microwave Device Operating near the Quantum Limit, Phys. Rev. Lett. 108, 147701 (2012).

[4] A. H. Myerson, D. J. Szwer, S. C. Webster, D. T. C. Allcock, M. J. Curtis, G. Imreh, J. A. Sherman, D. N. Stacey, A. M. 
Steane, and D. M. Lucas, High-Fidelity Readout of Trapped-Ion Qubits, Phys. Rev. Lett. 100, 200502 (2008).

[5] H. Paik, D. I. Schuster, L. S. Bishop, G. Kirchmair, G. Catelani, A. P. Sears, B. R. Johnson, M. J. Reagor, L. Frunzio, L. I. Glazman, S. M. Girvin, M. H. Devoret, and R.J. Schoelkopf, Observation of High Coherence in Josephson Junction Qubits Measured in a ThreeDimensional Circuit QED Architecture, Phys. Rev. Lett. 107, 240501 (2011).

[6] T. L. Nicholson, S. L. Campbell, R. B. Hutson, G. E. Marti, B. J. Bloom, R. L. McNally, W. Zhang, M. D. Barrett, M. S. Safronova, G. F. Strouse, W. L. Tew, and J. Ye, Systematic Evaluation of an Atomic Clock at $2 \times 10^{-18}$ Total Uncertainty, Nat. Commun. 6, 6896 (2015).

[7] P. W. Shor, in Proceedings of the IEEE Conference on Foundations of Computer Science (IEEE, New York, 1994), pp. 124-134.

[8] D. Gottesman, Stabilizer Codes and Quantum Error Correction, arXiv:quant-ph/9705052.

[9] A. Y. Kitaev, Fault-Tolerant Quantum Computation by Anyons, Ann. Phys. (Amsterdam) 303, 2 (2003).

[10] J. Cramer, N. Kalb, M. A. Rol, B. Hensen, M. S. Blok, M. Markham, D. J. Twitchen, R. Hanson, and T. H. Taminiau, Repeated Quantum Error Correction on a Continuously Encoded Qubit by Real-Time Feedback, Nat. Commun. 7, 11526 (2016).

[11] J. Kelly et al., State Preservation by Repetitive Error Detection in a Superconducting Quantum Circuit, Nature (London) 519, 66 (2015).

[12] N. Ofek, A. Petrenko, R. Heeres, P. Reinhold, Z. Leghtas, B. Vlastakis, Y. Liu, L. Frunzio, S. M. Girvin, L. Jiang, M. Mirrahimi, M. H. Devoret, and R. J. Schoelkopf, Demonstrating Quantum Error Correction that Extends the Lifetime of Quantum Information, arXiv:1602.04768.

[13] S. Lloyd, Universal Quantum Simulators, Science 273, 1073 (1996).

[14] S. Lloyd and L. Viola, Engineering Quantum Dynamics, Phys. Rev. A 65, 010101 (2001).

[15] D. Aharonov, A. Kitaev, and J. Preskill, Fault-Tolerant Quantum Computation with Long-Range Correlated Noise, Phys. Rev. Lett. 96, 050504 (2006).

[16] A. G. Fowler and J. M. Martinis, Quantifying the Effects of Local Many-Qubit Errors and Nonlocal Two-Qubit Errors on the Surface Code, Phys. Rev. A 89, 032316 (2014).

[17] J. M. Martinis, Qubit Metrology for Building a FaultTolerant Quantum Computer, Quantum Inf. doi:10.1038/ npjqi.2015.5 (2015).

[18] D. Ristè, M. P. da Silva, C. A. Ryan, A. W. Cross, J. A. Smolin, J. M. Gambetta, J. M. Chow, and B. R. Johnson, Demonstration of Quantum Advantage in Machine Learning, arXiv:1512.06069.

[19] M. Takita, A. D. Córcoles, E. Magesan, B. Abdo, M. Brink, A. Cross, J. M. Chow, and J. M. Gambetta, Demonstration of Weight-Four Parity Measurements in the Surface Code Architecture, arXiv:1605.01351.

[20] D. Nigg, M. Müller, E. A. Martinez, P. Schindler, M. Hennrich, T. Monz, M. A. Martin-Delgado, and R. Blatt, Quantum Computations on a Topologically Encoded Qubit, Science 345, 302 (2014).
[21] J. T. Barreiro, M. Müller, P. Schindler, D. Nigg, T. Monz, M. Chwalla, M. Hennrich, C. F. Roos, P. Zoller, and R. Blatt, An Open-System Quantum Simulator with Trapped Ions, Nature (London) 470, 486 (2011).

[22] J. M. Chow, J. M Gambetta, E. Magesan, D. W. Abraham, A. W. Cross, B. R. Johnson, N. A. Masluk, C. A. Ryan, J. A. Smolin, S. J. Srinivasan, and M. Steffen, Implementing a Strand of a Scalable Fault-Tolerant Quantum Computing Fabric, Nat. Commun. 5, 4015 (2014).

[23] O.-P. Saira, J. P. Groen, J. Cramer, M. Meretska, G. de Lange, and L. DiCarlo, Entanglement Genesis by AncillaBased Parity Measurement in 2D Circuit QED, Phys. Rev. Lett. 112, 070502 (2014).

[24] J. M. Gambetta, A. D. Córcoles, S. T. Merkel, B. R. Johnson, J. A. Smolin, J. M. Chow, C. A. Ryan, C. Rigetti, S. Poletto, T. A. Ohki, M. B. Ketchen, and M. Steffen, Characterization of Addressability by Simultaneous Randomized Benchmarking, Phys. Rev. Lett. 109, 240504 (2012).

[25] M. Dalmonte, S. I. Mirzaei, P. R. Muppalla, D. Marcos, P. Zoller, and G. Kirchmair, Realizing Dipolar Spin Models with Arrays of Superconducting Qubits, Phys. Rev. B 92, 174507 (2015).

[26] S. E. Nigg and S. M. Girvin, Stabilizer Quantum Error Correction Toolbox for Superconducting Qubits, Phys. Rev. Lett. 110, 243604 (2013).

[27] J. S. Lundeen, A. Feito, H. Coldenstrodt-Ronge, K. L. Pregnell, C. Silberhorn, T. C. Ralph, J. Eisert, M. B. Plenio, and I. A. Walmsley, Tomography of Quantum Detectors, Nat. Phys. 5, 27 (2009).

[28] M. A. Nielsen and I. L. Chuang, Quantum Computation and Quantum Information: 10th Anniversary Edition, 10th ed. (Cambridge University Press, New York, 2011).

[29] E. Knill, R. Laflamme, and G. J. Milburn, A Scheme for Efficient Quantum Computation with Linear Optics, Nature (London) 409, 46 (2001).

[30] P. Aliferis, D. Gottesman, and J. Preskill, Accuracy Threshold for Postselected Quantum Computation, Quantum Inf. Comput. 8, 181 (2008).

[31] E. Knill, Fault-Tolerant Postselected Quantum Computation: Schemes, arXiv:quant-ph/0402171.

[32] See Supplemental Material at http://link.aps.org/ supplemental/10.1103/PhysRevX.6.031041 for a description of the device and experimental apparatus, results of calibration experiments, theoretical treatment of the measurement analysis, and complete numerical results.

[33] M. Reagor, W. Pfaff, C. Axline, R. W. Heeres, N. Ofek, K. Sliwa, E. Holland, C. Wang, J. Z. Blumoff, K. Chou, M. J. Hatridge, L. Frunzio, M. H. Devoret, L. Jiang, and R. J. Schoelkopf, A Quantum Memory with Near-Millisecond Coherence in Circuit QED, Phys. Rev. B 94, 014506 (2016).

[34] C. Axline, C. Reagor, R. Heeres, P. Reinhold, C. Wang, K. Shain, W. Pfaff, Y. Chu, L. Frunzio, and R. J. Schoelkopf, A Coaxial Line Architecture for Integrating and Scaling 3D cQED Systems, Appl. Phys. Lett. 109, 042601 (2016).

[35] D. I. Schuster, A. A. Houck, J. A. Schreier, A. Wallraff, J. M. Gambetta, A. Blais, L. Frunzio, J. Majer, B. Johnson, M. H. Devoret, S. M. Girvin, and R. J. Schoelkopf, Resolving Photon Number States in a Superconducting Circuit, Nature (London) 445, 515 (2007). 
[36] B. Vlastakis, G. Kirchmair, Z. Leghtas, S. E. Nigg, L. Frunzio, S. M. Girvin, M. Mirrahimi, M. H. Devoret, and R. J. Schoelkopf, Deterministically Encoding Quantum Information Using 100-Photon Schrödinger Cat States, Science 342, 607 (2013).

[37] M. Wilde, From Classical to Quantum Shannon Theory, arXiv:1106.1445v7.

[38] M. Ozawa, Quantum Measuring Processes of Continuous Observables, J. Math. Phys. (N.Y.) 25, 79 (1984).

[39] E. B. Davies and J. T. Lewis, An Operational Approach to Quantum Probability, Commun. Math. Phys. 17, 239 (1970).

[40] A. Gilchrist, N. K. Langford, and M. A. Nielsen, Distance Measures to Compare Real and Ideal Quantum Processes, Phys. Rev. A 71, 062310 (2005).

[41] J. Dressel, T. A. Brun, and A. N. Korotkov, Implementing generalized measurements with superconducting qubits, Phys. Rev. A 90, 032302 (2014).

[42] E. Magesan and P. Cappellaro, Experimentally Efficient Methods for Estimating the Performance of Quantum Measurements, Phys. Rev. A 88, 022127 (2013).

[43] A. Y. Kitaev, Quantum Computations: Algorithms and Error Correction, Russ. Math. Surv. 52, 1191 (1997).

[44] L. Zhang, H. B. Coldenstrodt-Ronge, A. Datta, G. Puentes, J. S. Lundeen, X.-M. Jin, B. J. Smith, M. B. Plenio, and I. A. Walmsley, Mapping Coherence in Measurement via Full Quantum Tomography of a Hybrid Optical Detector, Nat. Photonics 6, 364 (2012).
[45] E. Knill, D. Leibfried, R. Reichle, J. Britton, R. B. Blakestad, J. D. Jost, C. Langer, R. Ozeri, S. Seidelin, and D. J. Wineland, Randomized Benchmarking of Quantum Gates, Phys. Rev. A 77, 012307 (2008).

[46] J. M. Chow, L. DiCarlo, J. M. Gambetta, A. Nunnenkamp, L. S. Bishop, L. Frunzio, M. H. Devoret, S. M. Girvin, and R. J. Schoelkopf, Detecting Highly Entangled States with a Joint Qubit Readout, Phys. Rev. A 81, 062325 (2010).

[47] M. Grant and S. Boyd, CVX: Matlab Software for Disciplined Convex Programming, Version 2.1, http://cvxr .com/cvx.

[48] M. Grant and S. Boyd, Graph Implementations for Nonsmooth Convex Programs (Springer-Verlag, Berlin/Heidelberg, 2008), pp. 95-110.

[49] R. Heeres et al. (to be published).

[50] N. Khaneja, T. Reiss, C. Kehlet, T. Schulte-Herbrüggen, and S. J. Glaser, Optimal Control of Coupled Spin Dynamics: Design of NMR Pulse Sequences by Gradient Ascent Algorithms, J. Magn. Reson. 172, 296 (2005).

[51] J. R. Johansson, P. D. Nation, and F. Nori, QuTiP 2: A Python Framework for the Dynamics of Open Quantum Systems, Comput. Phys. Commun. 184, 1234 (2013); see also http://qutip.org/.

[52] D. Gross, Y.-K. Liu, S. T. Flammia, S. Becker, and J. Eisert, Quantum State Tomography via Compressed Sensing, Phys. Rev. Lett. 105, 150401 (2010). 